\title{
Recent experiments at the JYFLTRAP Penning trap
}

\section{Anu Kankainen ${ }^{1}$ (D) . Tommi Eronen ${ }^{1}$ - Dmitrii Nesterenko ${ }^{1}$ - Antoine de Roubin ${ }^{1,2}$. Markus Vilén ${ }^{1,3}$}

Published online: 16 March 2020

(C) The Author(s) 2020

\begin{abstract}
The JYFLTRAP double Penning trap mass spectrometer at the Ion Guide Isotope Separator On-Line (IGISOL) facility offers excellent possibilities for high-precision mass measurements of radioactive ions. Around 400 atomic masses, including around 50 isomeric states, have been measured since JYFLTRAP became operational. JYFLTRAP has also been used as a high-resolution mass separator for decay spectroscopy experiments as well as an ion counter for fission yield studies. In this contribution, an overview of recent activities at the JYFLTRAP Penning trap is given, with a focus on nuclei discussed in the PLATAN2019 meeting.
\end{abstract}

Keywords Penning trap $\cdot$ Atomic mass $\cdot$ Nuclear binding energy $\cdot$ Isomers

\section{Introduction}

Atomic masses can be measured with the highest achievable precision using Penning trap mass spectrometers. Very high precision $\left(\leq 1 \mathrm{keV} / \mathrm{c}^{2}\right)$ mass measurements are important for fundamental physics $[1,2]$, such as for weak-interaction studies, testing the isospin symmetry or the unitarity of the Cabibbo-Kobayashi-Maskawa (CKM) quark mixing matrix. Mass measurements with a good precision of $\leq 10 \mathrm{keV} / \mathrm{c}^{2}$ are also needed to study subtle changes in nuclear structure $[2,3]$ or to provide accurate inputs for astrophysical reaction network calculations [4]. In this contribution, we review the recent activities at the JYFLTRAP double Penning trap mass spectrometer [5-7] located at the Ion Guide Isotope Separator

This article is part of the Topical Collection on Proceedings of PLATAN 2019, 1st International Conference, Merger of the Poznan Meeting on Lasers and Trapping Devices in Atomic Nuclei Research and the International Conference on Laser Probing, Mainz, Germany 19-24 May 2019

Edited by Krassimira Marinova, Michael Block, Klaus D.A. Wendt and Magdalena Kowalska

Anu Kankainen

anu.kankainen@jyu.fi

1 Department of Physics, University of Jyväskylä, P.O. Box 35 (YFL), FI-40014, Jyväskylä, Finland

2 Present address: Centre d'Etudes Nucléaires de Bordeaux Gradignan, Universite de Bordeaux, CENBG-CS 10120, F-33175 Gradignan Cedex, France

3 Present address: CERN, CH-1211, Geneva, Switzerland 


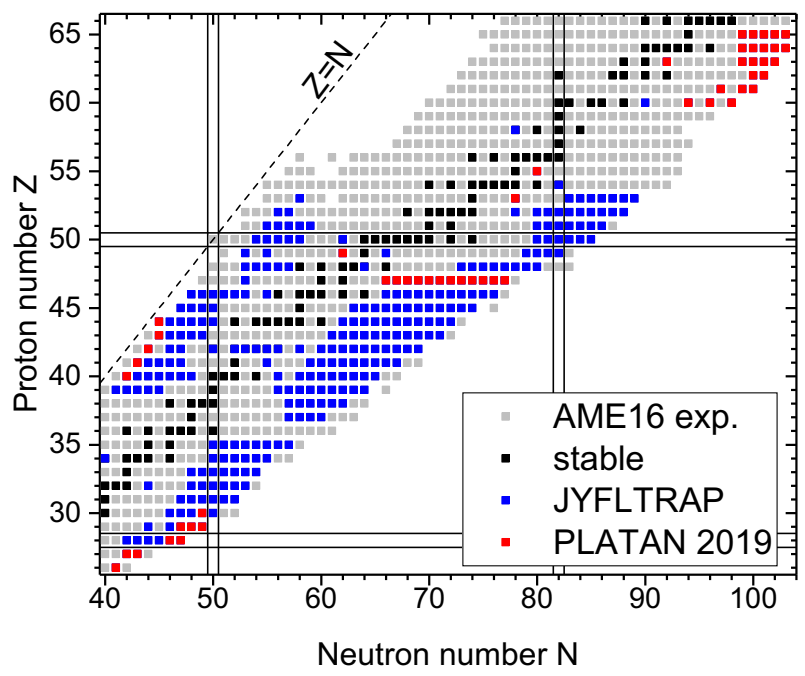

Fig. 1 JYFLTRAP measurements presented in PLATAN 2019 (in red) together with previous studies (in blue) for $Z=26-67$. Nuclei with experimental values in AME16 are also shown

On-Line (IGISOL) facility [8, 9] in the JYFL Accelerator Laboratory of the University of Jyväskylä, Finland.

The JYFLTRAP double Penning trap has been operational more than 15 years. During these years, around 400 atomic masses, including around 50 isomeric states, have been measured with JYFLTRAP. Most of the studied nuclei have been neutron-rich (around 2/3), produced by proton- or deuteron-induced fission on ${ }^{n a t} \mathrm{U}$ or Th targets. Around third of the measurements have been performed on the neutron-deficient side, where the ions of interest are produced via fusion-evaporation reactions. Figure 1 highlights in red the recent JYFLTRAP mass measurements presented in the PLATAN 2019 conference. What is not shown in Fig. 1, is that many of the measured nuclides have long-living isomeric states which have also been measured with JYFLTRAP. These provide important information on level structure far from stability. The recent ground and isomeric- state mass measurements at JYFLTRAP will be discussed in Section 3 and its subsections. JYFLTRAP has also been widely used for selecting isotopes or even isomers of interest for decay spectroscopy, or as an ion counter for fission yield studies. Recent post-trap and fission-yield publications will be shortly reviewed in Section. 4.

\section{JYFLTRAP double Penning trap at IGISOL}

JYFLTRAP [5-7] is a cylindrical double Penning trap mass spectrometer located inside a $7 \mathrm{~T}$ superconducting solenoid at the IGISOL facility [9]. The continuous radioactive ion beam from IGISOL is first mass-separated using a dipole magnet, which is usually sufficient to select the mass number of interest $A$. In addition to IGISOL, ion beams can be delivered from an offline ion source station [10], housing an electric discharge ion source and a surface ion source. There is also a space reservation for a laser ablation ion source.

The continuous ion beam is cooled and bunched in a radiofrequency quadrupole (RFQ) [11], and the ion bunches are further injected into the first trap of JYFLTRAP, also known 
as the preparation trap. In the first trap, the ions of interest are selected using the buffer-gas cooling technique [12], and sent further to the second trap for precision mass measurements, or to post-trap decay spectroscopy station after the traps. Usually the Time-of-Flight Ion Cyclotron Resonance (TOF-ICR) technique $[13,14]$ has been employed in the second trap, also known as the measurement trap, to determine the ion's cyclotron resonance frequency $v_{c}=\frac{1}{2 \pi} \frac{q}{m} B$, where $B$ is the magnetic field strength, and $q$ and $m$ are the charge and the mass of the ion, respectively. The magnetic field strength is calibrated by using reference ions with well-known mass values. A new technique to determine the ion's cyclotron resonance frequency, the Phase-Imaging Ion Cyclotron Resonance (PI-ICR) technique [15], was commissioned at JYFLTRAP in 2017, see Ref. [7]. A position-sensitive MCP ion detector with a delay-line anode (DLD40) from RoentDek GmbH [16] was installed behind the traps, and the extraction optics was modified accordingly. Since then, the new technique has been increasingly utilized for high-precision mass measurements at JYFLTRAP. The method gives consistent results with the conventional TOF-ICR technique, as demonstrated with the $Q$-value measurement for the neutrinoless double electron-capture on ${ }^{102} \mathrm{Pd}$ [17], but a higher precision can be achieved with the PI-ICR technique. Further examples of TOF-ICR and PI-ICR measurements are given in Section. 3.

\section{Recent mass measurements with JYFLTRAP}

Since the last LASER meeting held in Poznan in May 2016, several publications have come out from JYFLTRAP experiments. The $Q_{E C}$ value of the superallowed $\beta^{+}$emitter ${ }^{42} \mathrm{Sc}$ has been measured to be 6426.350(53) $\mathrm{keV} \mathrm{[18],} \mathrm{the} \mathrm{isobaric} \mathrm{multiplet} \mathrm{mass} \mathrm{equation} \mathrm{studied}$ for the quintet at $A=52$ [19] and a general review on ion traps given in [3]. These were already discussed in Poznan. Here we present recent activities at JYFLTRAP with a focus on the PLATAN2019 contributions.

\subsection{Nuclei in the vicinity of ${ }^{78} \mathrm{Ni}$}

Masses of nuclei close to ${ }^{78} \mathrm{Ni}$ are important for several reasons. Firstly, the evolution of the $Z=28$ and $N=50$ shell closures and the magicity of ${ }^{78} \mathrm{Ni}$ can be probed via mass measurements. Secondly, the masses of neutron-rich nuclei close to $N=50$, i.e. the strength of the $N=50$ shell closure, are relevant to understand in detail the core collapse phase of supernovae where electron captures on nuclei play a key role [20, 21]. In a recent JYFLTRAP experiment, several neutron-rich $\mathrm{Ni}, \mathrm{Cu}$ and $\mathrm{Zn}$ isotopes were measured to study these phenomena [22].

We also investigated nuclides below ${ }^{68} \mathrm{Ni}(Z=28, N=40)$, namely ${ }^{69,70} \mathrm{Co}$ and ${ }^{67} \mathrm{Fe}$. ${ }^{68} \mathrm{Ni}$ has some doubly magic features, such as high excitation energy of the first $2^{+}$state and low transition strength $B\left(E 2 ; 0_{g . s .} \rightarrow 2_{1}^{+}\right)[23,24]$. Previous mass measurements, however, have not shown a strong subshell closure at $N=40$ in the region [25-29]. The new JYFLTRAP measurements support the conclusion that the $N=40$ subshell closure is rather weak, and gets weaker below nickel $[30,31]$. The identification of the isomeric states in the cobalt isotopes is crucial for accurate empirical shell-gap energies and also for the investigation of a possible island of inversion below ${ }^{68} \mathrm{Ni}[32,33]$. At JYFLTRAP, only one state was observed for ${ }^{70} \mathrm{Co}$, whereas for ${ }^{69} \mathrm{Co}$ the data indicate that there are two states present.

The mass measurements of ${ }^{69} \mathrm{Co}$ and ${ }^{67} \mathrm{Fe}$ are also important for accurate neutron separation energies needed to calculate neutron-capture and their inverse photodisintegration 
rates in the astrophysical rapid neutron capture process ( $r$ process) $[34,35]$. Sensitivity studies [36] have shown that ${ }^{67} \mathrm{Fe}(n, \gamma){ }^{68} \mathrm{Fe}$ and ${ }^{68} \mathrm{Co}(n, \gamma){ }^{69} \mathrm{Co}$ have a strong impact on the calculated $r$-process abundances. With the new JYFLTRAP measurements, we could reduce the mass-related uncertainties in these impactful neutron-capture rates. Moreover, the photodisintegration rates are much higher than previously considered.

\subsection{Neutron-rich silver isotopes}

Nuclei close to the doubly magic ${ }^{132} \mathrm{Sn}$ are important both for nuclear structure and for the $r$-process. Mass measurements in this region provide information on the evolution of the $Z=50$ and $N=82$ shell closures, one- and two-neutron separation energies and pairing effects in the region. Recent studies have shown that the masses of the nuclei close to ${ }^{132} \mathrm{Sn}$ have the highest impact on the calculated $r$-process abundances for different astrophysical scenarios [37].

Recently neutron-rich silver isotopes ${ }^{113-124} \mathrm{Ag}$ were investigated at JYFLTRAP. Previously, ${ }^{112,114-124} \mathrm{Ag}$ have been studied at the ISOLTRAP Penning trap at CERN [38] and ${ }^{125,126} \mathrm{Ag}$ at the ESR storage ring at GSI [39]. Of these, the ESR measurements have uncertainties of 200-300 keV which are not adequate for the $r$-process modeling or detailed studies of nuclear structure. In addition, the ${ }^{126} \mathrm{Ag}$ mass obtained at ESR is $730(370) \mathrm{keV}$ lower than the extrapolated value in the Atomic Mass Evaluation 2016 [40]. The ISOLTRAP measurements using the TOF-ICR technique were hampered by the existence of low-lying isomeric states and difficulties to identify the measured state. In these measurements the state for ${ }^{115} \mathrm{Ag}$ and ${ }^{119} \mathrm{Ag}$ could not be assigned as the ground state or isomer, and ${ }^{121-124} \mathrm{Ag}$ were assumed to be an admixture of the states which increased the uncertainty of the measurements. ${ }^{120} \mathrm{Ag}$ was assigned as the ground state in [38], however, a decay spectroscopy study performed at Holifield Radioactive Ion Beam Facility (HRIBF) showed that ${ }^{120} \mathrm{Ag}$ actually has three long-living ( $>10 \mathrm{~ms}$ ) states [41]. Therefore, it is unclear which state was measured at ISOLTRAP, the ground state or the first isomeric state.

The mass measurements of silver isotopes at JYFLTRAP were performed with the PIICR technique $[7,15]$. Phase accumulation times in the measurement trap had to be selected for each case individually depending on the excitation energies of the isomers to fully separate them. Stable ${ }^{133} \mathrm{Cs}^{+}$ions were used for the calibration of the magnetic field. The measured states in the studied silver isotopes are shown in Table 1.

The excitation energies of the ${ }^{119 m, 120 m, 122 n, 123 m, 124 m} \mathrm{Ag}$ isomeric states were measured for the first time. The precision for several ground-state mass values was improved, since the isomeric states were separated in most of the cases. In ${ }^{121} \mathrm{Ag}$ only one state was observed, while the low-lying isomeric state is additionally known in literature [42]. In ${ }^{122} \mathrm{Ag}$ two states with different half-lives were observed, while three long-living states are known in literature [42]. The ground state and the first isomeric state with an unknown excitation energy in ${ }^{122} \mathrm{Ag}$ have similar half-lives and could not be distinguished. The excitation energies of the isomeric states in ${ }^{113-118} \mathrm{Ag}$ are known with a good accuracy (sub-keV) from spectroscopic measurements [42] and can be used to cross-check our mass measurements. Figure 2 shows the projection of ion cyclotron motion in the measurement trap onto the position-sensitive MCP detector for one of the PI-ICR measurements of ${ }^{120} \mathrm{Ag}$.

\subsection{Neutron-rich rare-earth isotopes}

Neutron-rich rare-earth isotopes have been studied in two recent mass measurement campaigns at JYFLTRAP [43, 44]. Altogether 22 nuclides have been measured, of which 14 
Table 1 States in silver isotopes studied at JYFLTRAP and their properties

\begin{tabular}{|c|c|c|c|}
\hline State & $J^{\pi}$ & $T_{1 / 2}$ & $E^{*}, \mathrm{keV}$ \\
\hline${ }^{113 g} \mathrm{Ag}$ & $1 / 2^{-}$ & $5.37(5) \mathrm{h}$ & \\
\hline${ }^{113 m} \mathrm{Ag}$ & $7 / 2^{+}$ & $68.7(16) \mathrm{s}$ & $43.5(1)$ \\
\hline${ }^{114 g} \mathrm{Ag}$ & $1^{+}$ & $4.6(1) \mathrm{s}$ & \\
\hline${ }^{115 g} \mathrm{Ag}$ & $1 / 2^{-}$ & $20.0(5) \mathrm{m}$ & \\
\hline${ }^{115 m} \mathrm{Ag}$ & $7 / 2^{+}$ & $18.0(7) \mathrm{s}$ & $41.16(10)$ \\
\hline${ }^{116 g} \mathrm{Ag}$ & $\left(0^{-}\right)$ & $3.83(8) \mathrm{m}$ & \\
\hline${ }^{116 m} \mathrm{Ag}$ & $\left(3^{+}\right)$ & $20(1) \mathrm{s}$ & $47.90(10)$ \\
\hline${ }^{116 n} \mathrm{Ag}$ & $\left(6^{-}\right)$ & $9.3(3) \mathrm{s}$ & $129.8(22)$ \\
\hline${ }^{117 g} \mathrm{Ag}$ & $1 / 2^{-} \#$ & $73.6(14) \mathrm{s}$ & \\
\hline${ }^{117 m} \mathrm{Ag}$ & $7 / 2^{+} \#$ & $5.34(5) \mathrm{s}$ & $28.6(2)$ \\
\hline${ }^{118 g} \mathrm{Ag}$ & $1^{-}$ & $3.76(15) \mathrm{s}$ & \\
\hline${ }^{118 n} \mathrm{Ag}$ & $4\left(^{+}\right)$ & $2.0(2) \mathrm{s}$ & $127.63(10)$ \\
\hline${ }^{119 g} \mathrm{Ag}$ & $1 / 2^{-} \#$ & $6.0(5) \mathrm{s}$ & \\
\hline${ }^{119 n} \mathrm{Ag}$ & $7 / 2^{+} \#$ & $2.1(1) \mathrm{s}$ & $20 \#(20 \#)$ \\
\hline${ }^{120 g} \mathrm{Ag}$ & $4\left(^{+}\right)$ & $1.52(7) \mathrm{s}$ & \\
\hline${ }^{120 m} \mathrm{Ag}$ & $\left(0^{-}, 1^{-}\right)$ & $940(100) \mathrm{ms}$ & $0 \#(50 \#)$ \\
\hline${ }^{120 n} \mathrm{Ag}$ & $7\left(^{-}\right)$ & $384(22) \mathrm{ms}$ & $203.0(2)$ \\
\hline${ }^{121 x} \mathrm{Ag}$ & $\mathrm{g}: 7 / 2^{+} \# ; \mathrm{m}: 1 / 2^{-} \#$ & $\mathrm{~g}: 780(20) \mathrm{ms} ; \mathrm{m}: 200 \# \mathrm{~ms}$ & $\mathrm{~m}: 20 \#(20 \#)$ \\
\hline${ }^{122 x} \mathrm{Ag}$ & $\mathrm{g}:\left(3^{+}\right) ; \mathrm{m}:\left(1^{-}\right)$ & $\mathrm{g}: 529(13) \mathrm{ms} ; \mathrm{m}: 550(50) \mathrm{ms}$ & $\mathrm{m}: 80 \#(50 \#)$ \\
\hline${ }^{122 n} \mathrm{Ag}$ & $9\left(^{-}\right)$ & $200(50) \mathrm{ms}$ & $80 \#(50 \#)$ \\
\hline${ }^{123 g} \mathrm{Ag}$ & $7 / 2^{+} \#$ & $300(5) \mathrm{ms}$ & \\
\hline${ }^{123 m} \mathrm{Ag}$ & $1 / 2^{-} \#$ & $100 \# \mathrm{~ms}$ & $20 \#(20 \#)$ \\
\hline${ }^{124 g} \mathrm{Ag}$ & $\left(2^{-}\right)$ & $177.9(26) \mathrm{ms}$ & \\
\hline${ }^{124 m} \mathrm{Ag}$ & $\left(8^{-}\right)$ & $144(20) \mathrm{ms}$ & $0 \#(100 \#)$ \\
\hline
\end{tabular}

$J^{\pi}, T_{1 / 2}$ and $E^{*}$ are the spin with the parity, the half-life and the excitation energy of the isomeric state, correspondingly, taken from NUBASE2016 [42]. The values estimated from systematic trends in neighboring nuclides are marked by \#. The ground states are indicated with ${ }^{g}$, the first isomeric state and the second isomeric state are indicated with ${ }^{m}$ and ${ }^{n}$, correspondingly. The states, where the ground state and the isomeric state could not be distinguished, are referred with ${ }^{x}$

for the first time. These include the first measurements of ${ }^{158} \mathrm{Nd},{ }^{160,161} \mathrm{Pm},{ }^{162,163} \mathrm{Sm}$, ${ }^{164,165} \mathrm{Eu},{ }^{164-167} \mathrm{Gd}$, and ${ }^{165,167,168} \mathrm{~Tb}$. The new mass values agree with the extrapolations of AME16 [40] in most of the cases. Typically, the JYFLTRAP values were somewhat higher than predicted by the extrapolations [44]. The largest deviations between the new JYFLTRAP mass values and AME16 were found for ${ }^{154} \mathrm{Nd}, 220(60) \mathrm{keV}$, and ${ }^{156} \mathrm{Nd}$, 260(200) keV. Both mass values have been previously based on beta-decay end-point energies $[45,46]$ which tend to underestimate the $\mathrm{Q}$ values, and thus the masses. Indeed, the JYFLTRAP mass value for ${ }^{154} \mathrm{Nd}$ agrees with the recent mass value from the Canadian Penning Trap (CPT) [47].

In the latter experimental campaign, the masses of ${ }^{162} \mathrm{Eu}$ and ${ }^{163} \mathrm{Gd}$ were remeasured using the TOF-ICR technique with a $1600 \mathrm{~ms}$ quadrupolar excitation time in the second trap 


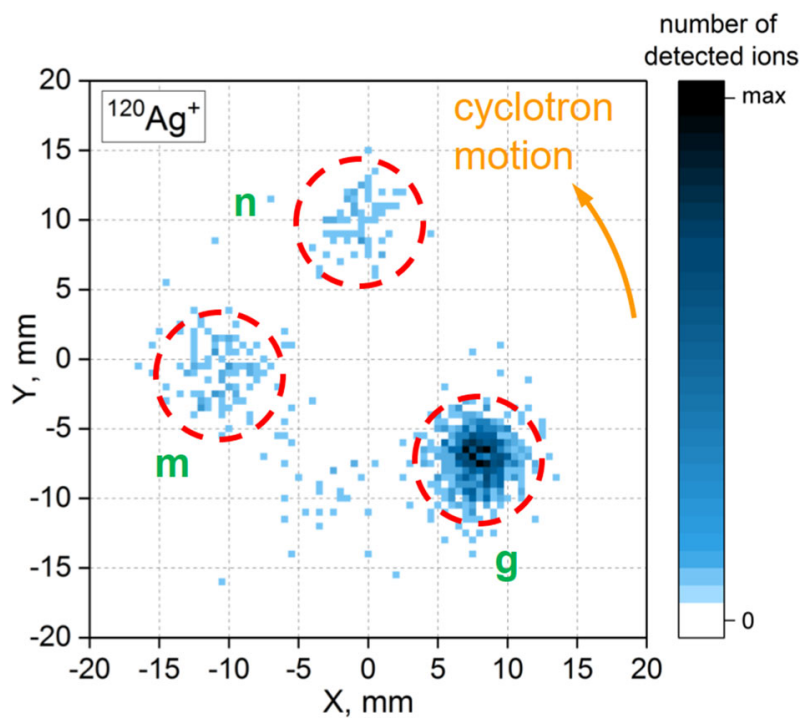

Fig. 2 Projection of the cyclotron motion of ${ }^{120} \mathrm{Ag}^{+}$ions onto the position-sensitive detector in PI-ICR method. The three detected ion spots on the detector correspond to the three long-living states in ${ }^{120} \mathrm{Ag}$

to resolve the ground and isomeric states from each other. In addition, the PI-ICR technique was applied for a detailed study of the ground and isomeric states in ${ }^{162} \mathrm{Eu}$, see Ref. [44].

The rare-earth masses are important for understanding the formation of the rare-earth abundance peak at $A=165$ in the $r$ process. It has been proposed to form via fission cycling [48] or during the freezeout when matter is decaying toward the stability. In the latter scenario, a kink in the neutron separation energies could funnel the flow toward the midshell $[49,50]$. Spectroscopic data indicate that there is an onset of deformation at $N=88-90$ : the $2^{+}$energies drop dramatically and the $E\left(4^{+}\right) / E\left(2^{+}\right)$ratios increase suddenly. Moreover, it has been suggested that there would be a subshell closure at $N=100$ based on the observed small kink in the $2^{+}$energies at $N=100$. In the JYFLTRAP campaign we wanted to investigate whether there is a kink in one- or two-neutron separation energies that could funnel the $r$-process flow or would support a subshell closure at $N=100$.

The new JYFLTRAP data do not introduce significant changes in the trends of twoneutron separation energies (see Fig. 3). No significant kinks supporting the proposed subshell closure or a change in the nuclear structure, are observed for the studied isotopic chains. Interestingly, neutron pairing energies were found to be lower than predicted by the commonly used theoretical models when approaching the midshell at $N=104$ [43, 44].

The impact of the JYFLTRAP mass values on the calculated $r$-process abundances was studied for a representative dynamical ejecta trajectory for a 1.35 solar-mass neutron-star merger from Ref. [51], with a very low initial electron fraction $Y_{e}=0.016$ and low entropy per baryon $s / k_{B}=8$. A simple asymmetric split [52] was assumed for fission fragment distributions to ensure that the rare-earth peak forms entirely via the dynamical formation mechanism of Refs. [49, 50]. Compared to a baseline study employing experimental mass values from AME16 [40] and theoretical mass values from the Finite-Range Droplet Model 


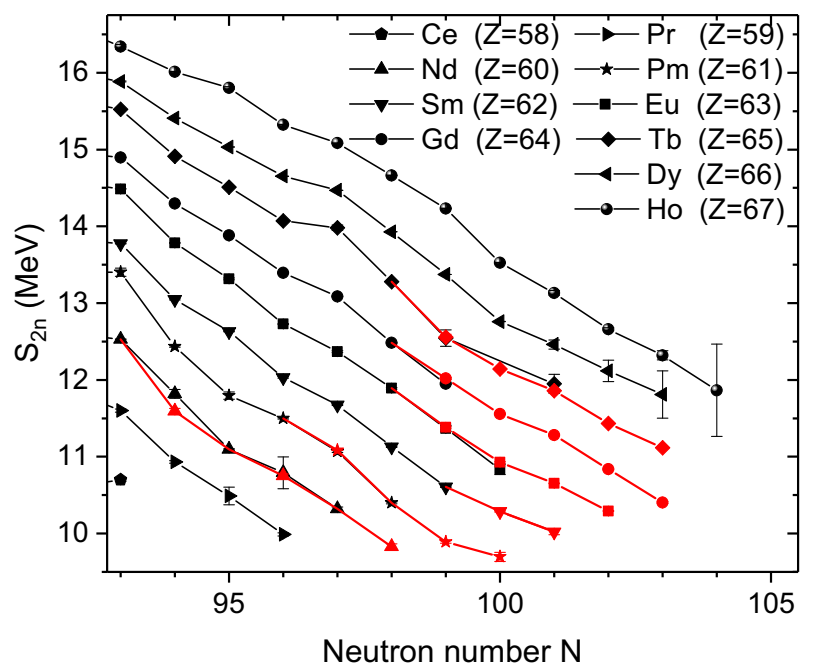

Fig. 3 Two-neutron separation energies for the studied isotopic chains in the rare-earth region. The black lines are based on AME16 values [40], and the values affected by the new JYFLTRAP measurements are highlighted in red

2012 (FRDM2012) [53], the new JYFLTRAP values produce a smoother abundance pattern and a better agreement with the observed $r$-process abundances is achieved [43, 44].

\subsection{Q-value measurements of rare weak decays}

Decays through weak interaction offer a possibility to determine the mass of a neutrino. In ordinary $\beta$ decays, the mass of a neutrino manifests itself as a distorted shape of the emitted beta spectrum close to the endpoint energy (i.e., $Q$-value). Such an experiment is KATRIN that uses tritium, whose $\beta$ endpoint energy is $18.6 \mathrm{keV} \mathrm{[54].} \mathrm{To} \mathrm{detect} \mathrm{a} \mathrm{distortion} \mathrm{in} \mathrm{the} \mathrm{beta}$ spectrum near the end-point energy, a $Q$-value as small as possible is desirable. This has prompted a survey to map rare weak decays $[55,56]$ that are $\beta^{+}, \beta^{-}$or EC decays of parent ground states to excited states in the daughter nucleus with a small $Q$-value (i.e., $<1 \mathrm{keV}$ ). The shape of the beta spectrum near the endpoint is expected to be relatively simple. These decays are also suitable for theoretical modeling of atomic effects in nuclear decay.

Several candidates for rare weak decays have been identified, all with well-known excitation energies in the daughter nucleus. However, the uncertainties in the ground-stateto-ground-state $Q$-values remain too high to extract a precise $Q$-value for a decay to an excited state. A direct parent-daughter mass ratio measurement with a relative precision of around $10^{-9}$ would allow extraction of $Q$-values at the uncertainty level of a few hundred $\mathrm{eV}$. It is imperative to know whether the $Q$-value is positive to find out the decays that could be feasible for determining the mass of a neutrino.

At JYFLTRAP, $Q$-values of four potential rare weak $\beta$-decay candidates were measured recently, summarized in Table 2 . As the nuclei of interest are close to stability, they were produced relatively easily. Out of the studied nuclei, ${ }^{135} \mathrm{Cs}$ was produced using proton-induced fission of natural uranium while the others were produced using proton- or deuteron-induced fusion reactions. The analysis of the collected data is ongoing. 
Table 2 Cases, whose ground-state-to-ground-state $Q$-values were measured at JYFLTRAP

\begin{tabular}{llll}
\hline Parent $\left(J^{\pi}\right)$ & Daughter $\left(J^{\pi}\right)$ & $E^{*}, \mathrm{keV}$ & $Q, \mathrm{keV}$ \\
\hline${ }^{111} \mathrm{In}\left(9 / 2^{+}\right)$ & ${ }^{111} \mathrm{Cd}\left(7 / 2^{+}\right)$ & $853.94 \pm 0.07$ & $6.36 \pm 3.0$ \\
& ${ }^{111} \mathrm{Cd}\left(3 / 2^{+}\right)$ & $855.6 \pm 1.0$ & $4.6 \pm 3.2$ \\
& ${ }^{111} \mathrm{Cd}\left(3 / 2^{+}\right)$ & $864.8 \pm 0.3$ & $-4.5 \pm 3.0$ \\
${ }^{131} \mathrm{I}\left(7 / 2^{+}\right)$ & ${ }^{131} \mathrm{Xe}\left(9 / 2^{+}\right)$ & $971.22 \pm 0.13$ & $-0.4 \pm 0.6$ \\
${ }^{155} \mathrm{Eu}\left(5 / 2^{+}\right)$ & ${ }^{155} \mathrm{Gd}\left(9 / 2^{-}\right)$ & $251.7056 \pm 0.0010$ & $0.1 \pm 0.9$ \\
${ }^{135} \mathrm{Cs}\left(7 / 2^{+}\right)$ & ${ }^{135} \mathrm{Ba}\left(11 / 2^{-}\right)$ & $268.218 \pm 0.020$ & $0.7 \pm 1.0$ \\
\hline
\end{tabular}

The first column shows the decaying parent state, the second column the excited state in the daughter nucleus, third column the excitation energy of the excited state in the daughter and the fourth column the $Q$-value to the excited state in the daughter. The tabulated spins and parities $\left(J^{\pi}\right)$ of the states, as well as the excitation energies and $Q$-values are based on Refs. [42, 57]

\subsection{Nuclei close to the $N=Z$ line in the $A=80-90$ mass region}

Heavier neutron-deficient nuclei close to the $N=Z$ line have been recently studied using an upgraded version of the heavy-ion ion-guide, HIGISOL [58], at IGISOL. The first online experiment with the upgraded system employed $222-\mathrm{MeV}^{36} \mathrm{Ar}^{8+}$ ions impinging into $a^{\text {nat }} \mathrm{Ni}$ target [59]. High-precision mass measurements were performed with JYFLTRAP for ${ }^{82} \mathrm{Zr},{ }^{84} \mathrm{Nb},{ }^{86} \mathrm{Mo},{ }^{88} \mathrm{Tc},{ }^{88} \mathrm{Tc}^{m}$ and ${ }^{89} \mathrm{Ru}[59,60]$. Two of the masses, ${ }^{88} \mathrm{Tc}^{m}$ and ${ }^{89} \mathrm{Ru}$, were measured for the first time and the precisions of ${ }^{82} \mathrm{Zr},{ }^{84} \mathrm{Nb}$ and ${ }^{88} \mathrm{Tc}$ were improved significantly. Additionally, the mass of ${ }^{86}$ Mo given in AME16 [40] was verified. The effect of the new data on the mass surface was studied, and similar behaviour as reported in the literature [40] was observed.

${ }^{88}$ Tc was studied at JYFLTRAP already in 2008 [61] but at the time the isomeric state ${ }^{88} \mathrm{Tc}^{m}$ could not be resolved from the more abundant ground state. With the PI-ICR technique, the mass of the isomer could be determined for the first time. The isomer was measured against the ground state using the PI-ICR technique, yielding an excitation energy of $E_{x}=70.4(31) \mathrm{keV}$ [59]. The mass of the dominantly produced ground state was measured using the TOF-ICR technique, which together with the excitation energy yielded also a mass value for the isomer.

The order of the three lowest states in ${ }^{88} \mathrm{Tc}$ was studied based on the obtained excitation energy for the isomeric state, Weisskopf estimates for the three states, and available spectroscopic data from literature. The most likely energies and spin-parities of the first three states in ${ }^{88} \mathrm{Tc}$ are $\left(E_{x}, J^{\pi}\right)=\left(0 \mathrm{keV}, 2^{+}\right),\left(70.4 \mathrm{keV}, 6^{+}\right)$and $\left(95 \mathrm{keV}, 4^{+}\right)$. Shell-model calculations were also performed for comparison but those were highly sensitive to the used model space and interaction model (for details, see Ref. [59]).

In addition to the six atomic masses that were directly measured, the masses of ${ }^{82} \mathrm{Mo}$ and ${ }^{86} \mathrm{Ru}\left(T_{z}=-1\right)$ were determined using theoretical mirror displacement energies (MDEs) and the directly measured masses of their respective mirror partners ${ }^{82} \mathrm{Zr}$ and ${ }^{86} \mathrm{Mo}\left(T_{z}=\right.$ +1 ) [59]. The resulting mass-excess values predict more tightly bound nuclei than literature [40], by more than $500 \mathrm{keV}$, and reduce the uncertainties of the predicted masses.

The JYFLTRAP mass measurements of ${ }^{82} \mathrm{Zr}$ and ${ }^{84} \mathrm{Nb}$ showed that mass values measured at the CSRe storage ring [62] deviate from the corresponding Penning-trap measurements, CSRe results being typically around $20 \mathrm{keV}$ smaller. In order to investigate whether this is a more general feature, published results from the CSRe storage ring were gathered and compared to available Penning-trap results. A total of 17 nuclides were available for 
the comparison. The deviation turned out to be larger than what would be expected based on statistical fluctuations alone [59].

\section{Other activities at JYFLTRAP}

In addition to precision mass measurements, JYFLTRAP has been employed in many posttrap decay spectroscopy experiments. Either the mass-selective buffer gas cooling technique [12] in the first trap, or dipolar Ramsey cleaning [63] in the second trap, has been used to select the isotope, or even the isomer of interest for spectroscopic studies after the trap. Monoisotopic or isomeric beams are beneficial for decay experiments as the background coming from the decays of neighbouring isobars is removed. Only daughter activities will be present but those can be reduced by implanting the ions into a tape which is regularly moved using a tape station.

Since the last LASER meeting held in May 2016 in Poznan, several trap-assisted spectroscopy studies employing JYFLTRAP have been published. A beta detector and an array of broad-energy range germanium detectors have been used to study the beta decays of ${ }^{88} \mathrm{Se}$ [64] and ${ }^{117} \mathrm{Rh}[65]$. The TASISpec silicon detector array together with a cluster and clover detectors were employed to study beta decay of ${ }^{127} \mathrm{Cd}$ [66]. Total absorption gamma-ray spectroscopy has been performed on the beta decays of ${ }^{87} \mathrm{Br},{ }^{88} \mathrm{Br}$, and ${ }^{94} \mathrm{Rb}$ [67], ${ }^{100} \mathrm{Tc}$ [68] and ${ }^{100 g s, 100 m} \mathrm{Nb}$ and ${ }^{102 g s, 102 m} \mathrm{Nb}$ [69]. The study of the first multiple-beta-delayed neutron emitter above $A=100,{ }^{136} \mathrm{Sb}$ [70], also involved JYFLTRAP to select only the ${ }^{136} \mathrm{Sb}$ ions for the studies.

JYFLTRAP has also turned out to be a great instrument to determine independent fission yields [71]. Neutron-rich isotopes at IGISOL are typically produced by proton-induced fission on ${ }^{n a t} \mathrm{U}$ or ${ }^{232} \mathrm{Th}$ target. The yields can be determined based on the quadrupole excitation scans performed in the first trap [12]. If the isomeric excitation energy is high enough, even independent isomeric yield ratios can be obtained from these studies. The results for isomeric yield ratios for ${ }^{81} \mathrm{Ge},{ }^{96,97} \mathrm{Y},{ }^{128,130} \mathrm{Sn}$, and ${ }^{129} \mathrm{Sb}$ in proton-induced fission on ${ }^{n a t} \mathrm{U}$ and ${ }^{232} \mathrm{Th}$ at $25 \mathrm{MeV}$ were reported in [72]. The new PI-ICR technique was recently employed for isomeric fission yield studies of ${ }^{119-127} \mathrm{Cd}$ and ${ }^{119-127} \mathrm{In}$ for $25-\mathrm{MeV}$ proton-induced fission on ${ }^{n a t} \mathrm{U}$ [73]. The benefit in the PI-ICR technique is that it has a superior resolving power as compared to the buffer-gas cooling technique in the first-trap as demonstrated in Fig. 1 of Ref. [73]. Hence, even low-lying isomeric states ( $\left.E_{x} \leq 100 \mathrm{keV}\right)$ and their fission yields can be studied. Moreover, there is no need to scan over a range of excitation frequencies. Every ion directly contributes to the yield measurement.

\section{Summary and outlook}

The scientific programme at JYFLTRAP is versatile, ranging from nuclear structure and astrophysics to experiments for neutrino physics or testing fundamental physics, such as isospin symmetry or the unitarity of the CKM quark mixing matrix. It is also used as an ion counter for fission yield studies and high-resolution mass separator for decay spectroscopy experiments. The recently commissioned PI-ICR technique has opened new possibilities to resolve and measure very low-lying isomeric states, as well as to count ions for isomeric fission yield studies. A multi-reflection time-of-flight mass spectrometer (MR-TOF) is under commission at JYFLTRAP. The MR-TOF will provide a faster method for mass measurements and help in reducing the isobaric background for the Penning-trap mass 
measurements. Combined with the broad range of radioactive nuclides produced at IGISOL, the prospects for high-precision mass measurements at JYFLTRAP look very promising in the future.

Acknowledgments Open access funding provided by University of Jyväskylä (JYU). This work has been supported by the Academy of Finland under the Finnish Centre of Excellence Programme 2012-2017 (Nuclear and Accelerator Based Physics Research at JYFL). A.K., D.N., T.E. and A.R. acknowledge support from the Academy of Finland under projects No. 275389, 284516, 312544, 295207, and 306980. This work was supported by the European Union's Horizon 2020 research and innovation program under grants No. 771036 (ERC CoG MAIDEN) and No. 654002 (ENSAR2). We thank for the bilateral mobility grant from the Institut Francais in Finland, the Embassy of France in Finland, the French Ministry of Higher Education and Research and the Finnish Society of Science and Letters. We are grateful for the mobility support from PICS MITICANS (Manipulation of Ions in Traps and Ion sourCes for Atomic and Nuclear Spectroscopy).

Open Access This article is licensed under a Creative Commons Attribution 4.0 International License, which permits use, sharing, adaptation, distribution and reproduction in any medium or format, as long as you give appropriate credit to the original author(s) and the source, provide a link to the Creative Commons licence, and indicate if changes were made. The images or other third party material in this article are included in the article's Creative Commons licence, unless indicated otherwise in a credit line to the material. If material is not included in the article's Creative Commons licence and your intended use is not permitted by statutory regulation or exceeds the permitted use, you will need to obtain permission directly from the copyright holder. To view a copy of this licence, visit http://creativecommonshorg/licenses/by/4.0/.

\section{References}

1. Blaum, K., Dilling, J., Nörtershäuser, W.: Precision atomic physics techniques for nuclear physics with radioactive beams. Phys. Scr. T152, 014017 (2013). https://doi.org/10.1088/0031-8949/2013/t152/ 014017

2. Dilling, J., Blaum, K., Brodeur, M., Eliseev, S.: Penning-Trap Mass Measurements in Atomic and Nuclear Physics. Annu. Rev. Nucl. Part. Sci. 68(1), 45 (2018). https://doi.org/10.1146/annurev-nucl102711-094939

3. Eronen, T., Kankainen, A., Äystö, J.: Ion traps in nuclear physics-Recent results and achievements. Progr. Part. Nucl. Phys. 91, 259 (2016). https://doi.org/10.1016/j.ppnp.2016.08.001. http://www.sciencedirect. com/science/article/pii/S0146641016300436

4. Schatz, H.: Nuclear masses in astrophysics. Int. J. Mass Spectrom. 349-350, 181 (2013). https://doi.org/10.1016/j.ijms.2013.03.016. http://www.sciencedirect.com/science/article/pii/S13873806 13001073. 100 years of Mass Spectrometry

5. Kolhinen, V., Kopecky, S., Eronen, T., Hager, U., Hakala, J., Huikari, J., Jokinen, A., Nieminen, A., Rinta-Antila, S., Szerypo, J., Äystö, J.: JYFLTRAP: a cylindrical Penning trap for isobaric beam purification at IGISOL. Nucl. Instrum. Meth. Phys. Res. Sect. A 528(3), 776 (2004). https://doi.org/10.1016/j.nima.2004.05.029. http://www.sciencedirect.com/science/article/pii/ S0168900204009854

6. Eronen, T., Kolhinen, V.S., Elomaa, V.V., Gorelov, D., Hager, U., Hakala, J., Jokinen, A., Kankainen, A., Karvonen, P., Kopecky, S., Moore, I.D., Penttilä, H., Rahaman, S., Rinta-Antila, S., Rissanen, J., Saastamoinen, A., Szerypo, J., Weber, C., Äystö, J.: JYFLTRAP: a Penning trap for precision mass spectroscopy and isobaric purification. Eur. Phys. J. A 48(4), 46 (2012). https://doi.org/10.1140/epja/i201212046-1

7. Nesterenko, D.A., Eronen, T., Kankainen, A., Canete, L., Jokinen, A., Moore, I.D., Penttilä, H., Rinta-Antila, S., de Roubin, A., Vilen, M.: Phase-Imaging Ion-Cyclotron-Resonance technique at the JYFLTRAP double Penning trap mass spectrometer. Eur. Phys. J. A 54(9), 154 (2018). https://doi.org/10.1140/epja/i2018-12589-y

8. Äystö, J.: Development and applications of the IGISOL technique. Phys, Nucl. A 693(1-2), 477 (2001). https://doi.org/10.1016/s0375-9474(01)00923-x 
9. Moore, I., Eronen, T., Gorelov, D., Hakala, J., Jokinen, A., Kankainen, A., Kolhinen, V., Koponen, J., Penttilä, H., Pohjalainen, I., Reponen, M., Rissanen, J., Saastamoinen, A., Rinta-Antila, S., Sonnenschein, V., Äystö, J.: Towards commissioning the new IGISOL-4 facility. Nucl. Instrum. Meth. Phys. Res. B 317, 208 (2013). https://doi.org/10.1016/j.nimb.2013.06.036

10. Vilén, M., Canete, L., Cheal, B., Giatzoglou, A., de Groote, R., de Roubin, A., Eronen, T., Geldhof, S., Jokinen, A., Kankainen, A., Moore, I., Nesterenko, D., Penttilä, H., Pohjalainen, I., Reponen, M., Rinta-Antila, S.: Mass measurements and production of ions at IGISOL for the astrophysical $r$ - and $r p$ processes. Nucl. Instrum. Meth. Phys. Res. Sect. B https://doi.org/10.1016/j.nimb.2019.04.051 http:// www.sciencedirect.com/science/article/pii/S0168583X19302344 (2019)

11. Nieminen, A., Huikari, J., Jokinen, A., Äystö, J., Campbell, P., Cochrane, E.: Beam cooler for low-energy radioactive ions. Nucl. Instrum. Meth. Phys. Res. Sect. A 469(2), 244 (2001). https://doi.org/10.1016/s0168-9002(00)00750-6

12. Savard, G., Becker, S., Bollen, G., Kluge, H.J., Moore, R., Otto, T., Schweikhard, L., Stolzenberg, H., Wiess, U.: A new cooling technique for heavy ions in a Penning trap. Phys. Lett. A 158(5), 247 (1991). http://www.sciencedirect.com/science/article/pii/0375960191910082

13. Gräff, G., Kalinowsky, H., Traut, J.: A direct determination of the proton electron mass ratio. Z. Phys. A 297(1), 35 (1980). https://doi.org/10.1007/bf01414243

14. König, M., Bollen, G., Kluge, H.J., Otto, T., Szerypo, J.: Quadrupole excitation of stored ion motion at the true cyclotron frequency. Int. J. Mass Spectrom. Ion Proc. 142(1-2), 95 (1995). https://doi.org/10. 1016/0168-1176(95)04146-c

15. Eliseev, S., Blaum, K., Block, M., Dörr, A., Droese, C., Eronen, T., Goncharov, M., Höcker, M., Ketter, J., Ramirez, E.M., Nesterenko, D.A., Novikov, Y.N., Schweikhard, L.: A phaseimaging technique for cyclotron-frequency measurements. Appl. Phys. B 114(1), 107 (2014). https://doi.org/10.1007/s00340-013-5621-0

16. http://www.roentdek.de. MCP delay line detector, RoentDek Handels GmbH

17. Nesterenko, D., Canete, L., Eronen, T., Jokinen, A., Kankainen, A., Novikov, Y., Rinta-Antila, S., de Roubin, A., Vilen, M.: High-precision measurement of the mass difference between ${ }^{102} \mathrm{Pd}$ and ${ }^{102} \mathrm{Ru}$. Int. J. Mass Spectrom. 435, 204 (2019). https://doi.org/10.1016/j.ijms.2018.10.038. http://www. sciencedirect.com/science/article/pii/S1387380618303257

18. Eronen, T., Hardy, J.C., Canete, L., Jokinen, A., Hakala, J., Kankainen, A., Kolhinen, V.S., Koponen, J., Moore, I.D., Murray, I.M., Penttilä, H., Pohjalainen, I., Poleshchuk, O., Reinikainen, J., Rinta-Antila, S., Soukouti, N., Voss, A., Äystö, J.: $Q_{\mathrm{EC}}$ value of the superallowed $\beta$ emitter ${ }^{42}$ Sc. Phys. Rev. C 95, 025501 (2017). https://doi.org/10.1103/PhysRevC.95.025501

19. Nesterenko, D.A., Kankainen, A., et al.: High-precision mass measurements for the isobaric multiplet mass equation at $A=52$. J. Phys. G: Nucl.Part. Phys. 44(6), 065103 (2017). https://doi.org/10.1088/13616471/aa67ae

20. Raduta, A.R., Gulminelli, F., Oertel, M.: Modification of magicity toward the dripline and its impact on electron-capture rates for stellar core collapse. Phys. Rev. C 93, 025803 (2016). https://doi.org/10.1103/PhysRevC.93.025803

21. Sullivan, C., O'Connor, E., Zegers, R.G.T., Grubb, T., Austin, S.M.: The sensitivity of core-collapse supernovae to nuclear electron capture. Astrophys. J. 816(1), 44 (2015). https://doi.org/10.3847/0004$637 \mathrm{x} / 816 / 1 / 44$

22. Giraud, S.: Mesures de masse autour du ${ }^{78} \mathrm{Ni}$ et nouveau traitement de l'équilibre statistique nucléaire pour l'étude des supernovae á effondrement de coeur. Ph.D. thesis Caen (2019)

23. Broda, R. et al.: $N=40$ Neutron Subshell Closure in the ${ }^{68} \mathrm{Ni}$ Nucleus. Phys. Rev. Lett. 74, 868 (1995). https://doi.org/10.1103/PhysRevLett.74.868

24. Sorlin, O., Leenhardt, S., Donzaud, C., Duprat, J., Azaiez, F., Nowacki, F., Grawe, H., Dombrádi, Z., et al.: ${ }_{28}^{68} \mathrm{Ni}_{40}$ : Magicity versus Superfluidity. Phys. Rev. Lett. 88, 092501 (2002). https://doi.org/10.1103/PhysRevLett.88.092501

25. Rahaman, S., Hakala, J., Elomaa, V.V., Eronen, T., Hager, U., Jokinen, A., Kankainen, A., Moore, I.D., Penttilä, H., Rinta-Antila, S., Rissanen, J., Saastamoinen, A., Weber, C., Äystö, J.: Masses of neutron-rich $\mathrm{Ni}$ and $\mathrm{Cu}$ isotopes and the shell closure at $\mathrm{Z}=28, \mathrm{~N}=40$. Eur. Phys. J. A 34(1), 5 (2007). https://doi.org/10.1140/epja/i2007-10489-y

26. Guénaut, C. et al.: High-precision mass measurements of nickel, copper, and gallium isotopes and the purported shell closure at $N=40$. Phys. Rev. C 75, 044303 (2007). https://doi.org/10.1103/PhysRevC. 75.044303

27. Block, M. et al.: Discovery of a Nuclear Isomer in ${ }^{65} \mathrm{Fe}$ with PenningTrap Mass Spectrometry. Phys. Rev. Lett. 100, 132501 (2008). https://doi.org/10.1103/PhysRevLett.100.132501 
28. Ferrer, R., Block, M., et al.: Penning trap mass spectrometry of neutron-rich Fe and Co isotopes around $N=40$ with the LEBIT mass spectrometer. Phys. Rev. C 81, 044318 (2010). https://doi.org/10.1103/PhysRevC.81.044318

29. Izzo, C. et al.: Precision mass measurements of neutron-rich Co isotopes beyond $N=40$. Phys. Rev. C 97, 014309 (2018). https://doi.org/10.1103/PhysRevC.97.014309

30. Canete, L., Giraud, S., Kankainen, A., Bastin, B., Nowacki, F., Poves, A., et al.: Precision mass measurements of ${ }^{67} \mathrm{Fe}$ and ${ }^{69,70} \mathrm{Co}$ : Nuclear structure toward $\mathrm{N}=40$ and impact on r-process reaction rates. Phys. Rev. C Rapid Comm. (2020)

31. Canete, L.: High precision mass measurements for nuclear astrophysics. Ph.D. thesis, Jyväskylä. http:// urn.fi/URN:ISBN:978-951-39-7693-4 (2019)

32. Nowacki, F., Poves, A., Caurier, E., Bounthong, B.: Shape Coexistence in ${ }^{78} \mathrm{Ni}$ as the Portal to the Fifth Island of Inversion. Phys. Rev. Lett. 117, 272501 (2016). https://doi.org/10.1103/ PhysRevLett.117.272501

33. Pauwels, D., Ivanov, O., Bree, N., Büscher, J., Cocolios, T.E., Gentens, J., Huyse, M., Korgul, A., Kudryavtsev, Y., Raabe, R., Sawicka, M., Stefanescu, I., Van de Walle, J., Van den Bergh, P., Van Duppen, P., Walters, W.B.: Shape isomerism at $N=40$ : Discovery of a proton intruder state in ${ }^{67}$ Co. Phys. Rev. C 78, 041307 (2008). https://doi.org/10.1103/PhysRevC.78.041307

34. Arnould, M., Goriely, S., Takahashi, K.: The r-process of stellar nucleosynthesis: Astrophysics and nuclear physics achievements and mysteries. Phys. Rep. 450(4-6), 97 (2007). https://doi.org/10.1016/j. physrep.2007.06.002

35. Horowitz, C.J. et al.: r-process nucleosynthesis: connecting rare-isotope beam facilities with the cosmos. J. Phys. G: Nucl. Part. Phys. 46(8), 083001 (2019). https://doi.org/10.1088/1361-6471/ab0849

36. Surman, R., Mumpower, M., Sinclair, R., Jones, K.L., Hix, W.R., McLaughlin, G.C.: Sensitivity studies for the weak $\mathrm{r}$ process: neutron capture rates. AIP Advances 4(4), 041008 (2014). https://doi.org/10.1063/1.4867191

37. Mumpower, M.R., Surman, R., Fang, D.L., Beard, M., Möller, P., Kawano, T., Aprahamian, A.: Impact of individual nuclear masses on $r$-process abundances. Phys. Rev. C 92, 035807 (2015). https://doi.org/10.1103/PhysRevC.92.035807

38. Breitenfeldt, M., Borgmann, C., et al.: Approaching the $N=82$ shell closure with mass measurements of Ag and Cd isotopes. Phys. Rev. C 81, 034313 (2010). https://doi.org/10.1103/PhysRevC.81.034313

39. Knöbel, R., Diwisch, M., Geissel, H., Litvinov, Y.A., Patyk, Z., Plaß, W.R., Scheidenberger, C., Sun, B., Weick, H., et al.: New results from isochronous mass measurements of neutron-rich uranium fission fragments with the FRS-ESR-facility at GSI. Eur. Phys. J. A 52(5), 138 (2016). https://doi.org/10.1140/epja/i2016-16138-6

40. Wang, M., Audi, G., Kondev, F., Huang, W., Naimi, S., Xu, X.: The AME2016 atomic mass evaluation (II). Tables, graphs and references. Chin. Phys. C 41(3), 030003 (2017). http://stacks.iop.org/1674-1137/ $41 / \mathrm{i}=3 / \mathrm{a}=030003$

41. Batchelder, J.C., Brewer, N.T., Goans, R.E., Grzywacz, R., Griffith, B.O., Jost, C., Korgul, A., Liu, S.H., Paulauskas, S.V., Spejewski, E.H., Stracener, D.W.: Low-lying collective states in ${ }^{120}$ Cd populated by $\beta$ decay of ${ }^{120} \mathrm{Ag}$ : Breakdown of the anharmonic vibrator model at the three-phonon level. Phys. Rev. C 86, 064311 (2012). https://doi.org/10.1103/PhysRevC.86.064311

42. Audi, G., Kondev, F.G., Wang, M., Huang, W., Naimi, S.: The NUBASE 2016 evaluation of nuclear properties. Chin. Phys. C 41(3), 030001 (2017). https://doi.org/10.1088/1674-1137/41/3/030001

43. Vilen, M., Kelly, J.M., Kankainen, A., Brodeur, M., et al.: Precision Mass Measurements on NeutronRich Rare-Earth Isotopes at JYFLTRAP: Reduced Neutron Pairing and Implications for $r$-Process Calculations. Phys. Rev. Lett. 120, 262701 (2018). https://doi.org/10.1103/PhysRevLett.120.262701

44. Vilén, M., Kelly, J.M., Kankainen, A., Brodeur, M., et al.: Exploring the mass surface near the rare-earth abundance peak via precision mass measurements at JYFLTRAP. Phys. Rev. C (2020)

45. Greenwood, R., Putnam, M.: Measurement of $\beta^{-}$end-point energies using a Ge detector with Monte Carlo generated response functions. Nucl. Instrum. Meth. Phys. Res. Sect. A 337(1), 106 (1993). https://doi.org/10.1016/0168-9002(93)91142-A. http://www.sciencedirect.com/science/article/ pii/016890029391142A

46. Shibata, M., Shindou, T., Kawade, K., Kojima, V., Taniguchi, A., Kawase, Y., Ichikawa, S. In: J. Äystö, Dendooven, P., Jokinen, A., Leino, M. (eds.): in Exotic Nuclei and Atomic Masses. Proceedings of the Third International Conference on Exotic Nuclei and Atomic Masses ENAM 2001 Hämeenlinna, Finland, 2-7 July 2001, p. 479. Springer, Berlin (2003). https://doi.org/10.1007/978-3-642-55560-2 ISBN: 978-3-540-00101-0

47. Orford, R. et al:: Precision mass measurements of neutron-rich neodymium and samarium isotopes and their role in understanding rare-earth peak formation. Phys. Rev. Lett. 120, 262702 (2018). https://doi.org/10.1103/PhysRevLett.120.262702 
48. Goriely, S., Sida, J.L., Lemaître, J.F., Panebianco, S., Dubray, N., Hilaire, S., Bauswein, A., Janka, H.T.: New Fission Fragment Distributions and $r$-Process Origin of the Rare-Earth Elements. Phys. Rev. Lett. 111, 242502 (2013). https://doi.org/10.1103/PhysRevLett.111.242502

49. Surman, R., Engel, J., Bennett, J.R., Meyer, B.S.: Source of the Rare-Earth Element Peak in $r$-Process Nucleosynthesis. Phys. Rev. Lett. 79, 1809 (1997). https://doi.org/10.1103/PhysRevLett.79.1809

50. Mumpower, M.R., McLaughlin, G.C., Surman, R.: Formation of the rare-earth peak: Gaining insight into late-time $r$-process dynamics. Phys. Rev. C 85, 045801 (2012). https://doi.org/10.1103/PhysRevC. 85.045801

51. Mendoza-Temis, J.D.J., Wu, M.R., Langanke, K., Martínez-Pinedo, G., Bauswein, A., Janka, H.T.: Nuclear robustness of the $r$ process in neutron-star mergers. Phys. Rev. C 92, 055805 (2015). https://doi.org/10.1103/PhysRevC.92.055805

52. Mumpower, M.R., McLaughlin, G.C., Surman, R., Steiner, A.W.: Reverse engineering nuclear properties rare-earth abundances in the r process. J. Phys. G: Nucl. Part. Phys. 44(3), 034003 (2017). https://doi.org/10.1088/1361-6471/44/3/034003

53. Möller, P., Sierk, A., Ichikawa, T., Sagawa, H.: Nuclear ground-state masses and deformations: FRDM(2012). At. Data Nucl. Data Tables 109-110, 1 (2016). https://doi.org/10.1016/j.adt.2015.10.002. http://www.sciencedirect.com/science/article/pii/S0092640X1600005X

54. Aker, M., Altenmüller, K., Arenz, M., Babutzka, M., Barrett, J., et al.: Improved Upper Limit on the Neutrino Mass from a Direct Kinematic Method by KATRIN. Phys. Rev. Lett. 123(22) https://doi.org/10.1103/physrevlett.123.221802 (2019)

55. Suhonen, J.: Theoretical studies of rare weak processes in nuclei. Phys. Scr. 89(5), 054032 (2014). http:// stacks.iop.org/1402-4896/89/i=5/a=054032

56. Gamage, N.D., Bhandari, R., Gamage, M.H., Sandler, R., Redshaw, M.: Identification and investigation of possible ultra-low $Q$-value $\beta$ decay candidates. Hyperfine Interact. 240(1), https://doi.org/10.1007/s10751-019-1588-5 (2019)

57. National Nuclear Data Center. https://www.nndc.bnl.gov/

58. Huikari, J., Dendooven, P., Jokinen, A., Nieminen, A., Penttilä, H., Peräjärvi, K., Popov, A., RintaAntila, S., Äystö, J.: Production of neutron-deficient rare isotope beams at IGISOL on-line and offline studies. Nucl. Instrum. Meth. Phys. Res. B 222(3-4), 632 (2004). https://doi.org/10.1016/j.nimb. 2004.04.164

59. Vilén, M., Kankainen, A., Baczyk, P., et al.: High-precision mass measurements and production of neutron-deficient isotopes using heavy-ion beams at IGISOL. Phys. Rev. C 100, 054333 (2019). https://doi.org/10.1103/PhysRevC.100.054333

60. Vilén, M.: Mass measurements and production of ions at IGISOL for the astrophysical $r$ - and $r p$-processes. Ph.D. thesis, Jyväskylä.. http://urn.fi/URN:ISBN:978-951-39-7838-9 (2019)

61. Weber, C., Elomaa, V.V., Ferrer, R., Fröhlich, C., et al.: Mass measurements in the vicinity of the $r p$ process and the $v p$-process paths with the Penning trap facilities JYFLTRAP and SHIPTRAP. Phys. Rev. C 78, 054310 (2008). https://doi.org/10.1103/PhysRevC.78.054310

62. Xing, Y. et al.: Mass measurements of neutron-deficient $\mathrm{Y}, \mathrm{Zr}$, and $\mathrm{Nb}$ isotopes and their impact on rp and vp nucleosynthesis processes. Phys. Lett. B 781, 358 (2018). https://doi.org/10.1016/j.physletb.2018. 04.009

63. Eronen, T., Elomaa, V.V., Hager, U., Hakala, J., Jokinen, A., Kankainen, A., Rahaman, S., Rissanen, J., Weber, C., Äystö, J.: Preparing isomerically pure beams of short-lived nuclei at JYFLTRAP. Nucl. Instrum. Meth. Phys. Res. B 266(19), 4527 (2008). https://doi.org/10.1016/j.nimb.2008.05.076. http:// www.sciencedirect.com/science/article/pii/S0168583X08007696. Proceedings of the XVth International Conference on Electromagnetic Isotope Separators and Techniques Related to their Applications

64. Czerwinski, M., Sieja, K., Rzaca-Urban, T., Urban, W., Płochocki, A., Kurpeta, J., Wisniewski, J., et al.: Penning-trap-assisted study of excitations in ${ }^{88} \mathrm{Br}$ populated in $\beta$ decay of ${ }^{88} \mathrm{Se}$. Phys. Rev. C 95, 024321 (2017). https://doi.org/10.1103/PhysRevC.95.024321

65. Kurpeta, J., Płochocki, A., Urban, W., Eronen, T., Jokinen, A., Kankainen, A., Kolhinen, V.S., Moore, I.D., Penttilä, H., Pomorski, M., Rinta-Antila, S., Rzaca-Urban, T., Wisniewski, J.: Excited levels in the multishaped ${ }^{117} \mathrm{Pd}$ nucleus studied via $\beta$ decay of ${ }^{117} \mathrm{Rh}$. Phys. Rev. C 98, 024318 (2018). https://doi.org/10.1103/PhysRevC.98.024318

66. Lorenz, C., Sarmiento, L.G., Rudolph, D., Golubev, P., et al.: $\beta$ decay of ${ }^{127} \mathrm{Cd}$ and excited states in ${ }^{127}$ In. Phys. Rev. C 99, 044310 (2019). https://doi.org/10.1103/PhysRevC.99.044310

67. Valencia, E., Tain, J.L., Algora, A., Agramunt, J., Estevez, E., et al.: Total absorption $\gamma$-ray spectroscopy of the $\beta$-delayed neutron emitters ${ }^{87} \mathrm{Br},{ }^{88} \mathrm{Br}$, and ${ }^{94} \mathrm{Rb}$. Phys. Rev. C 95, 024320 (2017). https://doi.org/10.1103/PhysRevC.95.024320

68. Guadilla, V., Algora, A., Tain, J.L., Agramunt, J., Jordan, D., Montaner-Pizá, A., Orrigo, S.E.A., Rubio, B., Valencia, E., Suhonen, J., Civitarese, O., Äystö, J., et al.: Experimental study of 
${ }^{100}$ Tc beta decay with total absorption $\gamma$-ray spectroscopy. Phys. Rev. C 96, 014319 (2017). https://doi.org/https://doi.org/10.1103/PhysRevC.96.014319

69. Guadilla, V., Algora, A., Tain, J.L., Estienne, M., Fallot, M., Sonzogni, A.A., et al.: Large Impact of the Decay of Niobium Isomers on the Reactor $\bar{v}_{e}$ Summation Calculations. Phys. Rev. Lett. 122, 042502 (2019). https://doi.org/10.1103/PhysRevLett.122.042502

70. Caballero-Folch, R., Dillmann, I., Agramunt, J., Taín, J.L., Algora, A., Äystö, J., et al.: First determination of $\beta$-delayed multiple neutron emission beyond $A=100$ through direct neutron measurement: The $P_{2 n}$ value of ${ }^{136} \mathrm{Sb}$. Phys. Rev. C 98, 034310 (2018). https://doi.org/10.1103/PhysRevC.98.034310

71. Penttilä, H., Elomaa, V.V., Eronen, T., Hakala, J., Jokinen, A., Kankainen, A., Moore, I.D., Rahaman, S., Rinta-Antila, S., Rissanen, J., Rubchenya, V., Saastamoinen, A., Weber, C., Äystö, J.: Fission yield studies at the IGISOL facility. Eur. Phys. J. A 48(4), 43 (2012). https://doi.org/10.1140/epja/i2012-12043-4

72. Rakopoulos, V., Lantz, M., Solders, A., Al-Adili, A., Mattera, A., et al.: First isomeric yield ratio measurements by direct ion counting and implications for the angular momentum of the primary fission fragments. Phys. Rev. C 98, 024612 (2018). https://doi.org/10.1103/PhysRevC.98.024612

73. Rakopoulos, V., Lantz, M., Pomp, S., Solders, A., Al-Adili, A., et al.: Isomeric fission yield ratios for odd-mass $\mathrm{Cd}$ and In isotopes using the phase-imaging ion-cyclotron-resonance technique. Phys. Rev. C 99, 014617 (2019). https://doi.org/10.1103/PhysRevC.99.014617

Publisher's note Springer Nature remains neutral with regard to jurisdictional claims in published maps and institutional affiliations. 